\title{
Editorial: Innovation and System Integration for Offshore Renewable Energy Structures
}

\author{
Dezhi Ning ${ }^{1 *}$, Lars Johanning ${ }^{2}$, Zhen $\mathrm{Gao}^{3}$, Philipp Rudolf Thies ${ }^{2}$, Wei Shi ${ }^{1}$ and \\ Kathleen Aviso ${ }^{4}$ \\ ${ }^{1}$ State Key Laboratory of Coastal and Offshore Engineering, Dalian University of Technology, Dalian, China, ${ }^{2}$ College of \\ Engineering, Mathematics and Physical Sciences, University of Exeter, Exeter, United Kingdom, ${ }^{3}$ Department of Marine \\ Technology, Norwegian University of Science and Technology, Trondheim, Norway, ${ }^{4}$ Chemical Engineering Department, De \\ La Salle University, Manila, Philippines
}

Keywords: offshore renewable energy, integration system, numerical modeling, experimental testing, software integration, new device concept

\section{Editorial on the Research Topic}

\section{Innovation and System Integration for Offshore Renewable Energy Structures}

Offshore Renewable Energy is an increasingly important field of ocean engineering research. The need for cost-effective yet reliable structures to withstand dynamic and extreme load conditions throughout the asset lifetime requires continuous innovation. The challenge to implement these innovations is to assess, demonstrate, and integrate the technologies and approaches into existing engineering practice.

This special issue on Innovation and System Integration for Offshore Renewable Energy Structures has solicited the latest research innovations in numerical modeling, experimental testing, software integration, and new device concepts for offshore renewable energy.

The editors are grateful to all contributing authors and reviewers, who enabled this special issue.

Wang et al. compute the loads on bottom-mounted circular cylinders to better estimate conditions for monopile foundations subjected to freak waves. Their results show that basic Morrison loads estimates are not conservative in these conditions.

Qiao et al. investigate design principles for oscillating wave energy to determine the appropriate scaling criteria along the energy conversion stage. The first stage energy conversion requires Froude similarity, whilst the second and third stage conversion can be replicated with power similarity relationships.

The cost and benefit of a novel wave energy converter design, encouraging marine growth on a biological frame to provide the structural mass, are explored by Hildebrand et al.. Their findings encourage the consideration of biological features for the design of wave energy converters in order to benefit from the inherent biodegradability and lower embedded carbon.

The enhanced prediction for the dynamic response of floating wind turbines is the subject of the paper by Chen et al.. They employ machine learning techniques to increase the numerical representation of the six degrees of freedom response in physical tank experiments, considerably decreasing the maximum error.

Deng et al. consider the performance of an asymmetric Oscillating Water Column device on top of a breakwater. Their OpenFOAM model demonstrates that coupling OWCs and breakwaters increase the wave frequency bandwidth of the system, increasing wave energy capture.

The collection of papers will assist researchers, designers, practitioners, and engineers to incorporate the latest innovations in offshore renewable energy structures. This issue truly illustrates that offshore engineering for renewable energy is a truly interdisciplinary research effort, bringing together coastal and civil engineering, computational modeling, software development, hydrodynamics, and marine biology. The editors trust the readers will find this collection interesting and look forward to further developments in this exciting field of research. 


\section{AUTHOR CONTRIBUTIONS}

DN and LJ: conceptualization, methodology, and writing-reviewing and editing. ZG: writingreviewing and editing. PT: writing original draft. WS and KA: writing-reviewing and editing. All authors contributed to the article and approved the submitted version.

Conflict of Interest: The authors declare that the research was conducted in the absence of any commercial or financial relationships that could be construed as a potential conflict of interest.
Publisher's Note: All claims expressed in this article are solely those of the authors and do not necessarily represent those of their affiliated organizations, or those of the publisher, the editors and the reviewers. Any product that may be evaluated in this article, or claim that may be made by its manufacturer, is not guaranteed or endorsed by the publisher.

Copyright (c) 2021 Ning, Johanning, Gao, Thies, Shi and Aviso. This is an open-access article distributed under the terms of the Creative Commons Attribution License (CC $B Y)$. The use, distribution or reproduction in other forums is permitted, provided the original author(s) and the copyright owner(s) are credited and that the original publication in this journal is cited, in accordance with accepted academic practice. No use, distribution or reproduction is permitted which does not comply with these terms. 